\title{
Spinal growth tethering: indications and limits
}

\author{
Peter O. Newton \\ Rady Children's Specialist of San Diego, San Diego, CA, USA \\ Correspondence to: Peter O. Newton, MD. Rady Children's Specialist of San Diego, 3020 Children's Way, MC5062, San Diego, CA 92123, USA. \\ Email: pnewton.rady@gmail.com
}

\begin{abstract}
The standard of care for progressive spinal deformity that is greater than 45-50 degrees in growing children is deformity correction with spinal fusion and instrumentation. This sacrifice both spinal motion and further spinal growth of the fused region. Idiopathic scoliosis in particular is associated with disproportionate anterior spinal column length compared to the posterior column (hypokyphosis) that is associated with the coronal (scoliosis) and axial plane (rib and lumbar prominence) deformities. In theory, application of compression to the convex and anterior aspects of vertebrae could decrease both anterior and lateral growth via the Hueter-Volkmann principle, while allowing growth on the concave and posterior aspect resulting in spinal realignment created by altered growth. Animal models and preliminary clinical experience suggest spinal growth can be modulated in this way using a flexible tether applied to the convex side of scoliotic vertebral column. Experimental studies suggest disc health is preserved with a flexible tether as disc motion is maintained during the growth period. Anterolateral tethering been performed via a thoracoscopic spinal approach clinically for a number of years and the early clinical outcomes are beginning to appear in the literature. Initial results of anterolateral tethering in growing patients with spinal deformities are encouraging, however the results 3-4 years after the procedure are somewhat mixed. Further research is ongoing and many remain optimistic that improvements in technology and understanding will continue to lead to better patient outcomes.
\end{abstract}

Keywords: Spinal growth modulation; vertebral growth modulation; anterolateral tether; fusionless scoliosis treatment; scoliosis treatment

Submitted Nov 18, 2019. Accepted for publication Dec 31, 2019.

doi: $10.21037 /$ atm.2019.12.159

View this article at: http://dx.doi.org/10.21037/atm.2019.12.159

\section{Introduction}

Spinal instrumentation and fusion remain the primary method of treatment for children with severe scoliosis. Although clinical outcome studies have demonstrated satisfactory results in terms of spinal deformity correction and long-term patient satisfaction, the effect of spinal fusion surgery is a permanent reduction in spinal motion and alteration on the loading on adjacent segments, and may lead to problems with later degeneration (1-6). These concerns have motivated investigators to study alternative treatment options that might preserve spinal motion, and maintain intervertebral disc health, while limiting or correcting the scoliotic deformity. The goal would be to provide a means for children to harness remaining spinal growth to produce correction. The ultimate goal of tethering is to create a more normal spinal contour while preserving functional motion. This summarizes the rationale of applying an anterolateral flexible tether as a growth modulation technique based on experimental studies and provides the clinical evidence to date regarding the application of this technology to treat childhood spinal deformities.

\section{Anterior spinal growth modulation}

Anterolateral vertebral body implants are now being used as an internal mechanical restraint to limit scoliosis progression, and ideally reduce the deformity by harnessing the patient's remaining spinal growth. Vertebral body 
tethering with a flexible cord can delay or eliminate the need for a definitive fusion procedure.

Early attempts to provide progressive scoliosis correction were attempted with vertebral body staples, applying the principles of long bone growth modulation, presented in 1951 by Nachlas and Borden (7). However, the vertebral staples were required to cross the intervertebral disc, and movement led to problematic loosening of the implants. Advancements in use of a temperature sensitive shape memory metal alloy (nitinol), have led to less loosening, but were only able modulate growth in moderate sized curves $(1,8-14)$.

\section{Animal models}

Braun et al. (15) reported on a variety of fusionless scoliosis implant strategies that were tested in the rat tail model. The results demonstrated that dynamic loading of the vertebrae provided the greatest growth modulation potential. Aronsson et al. similarly showed that alternating compression and distraction in the calf tail model also suggested that dynamic motion was be preferred (16).

Anterolateral spinal growth modulation has been tested in a variety of animal models. In 2002, Newton et al. (17) reported on eight immature calves instrumented with anterior vertebral body screws connected to a cable in the thoracic spine. Two screws were connected by a stainlesssteel tether and two were left unconnected to serve as a control. After 12 weeks of growth, coronal and sagittal plane deformities were consistently created over the tethered motion segments, compared to control segments. A followup study evaluating multilevel growth modulation in the bovine model found the flexible tethering system was able to consistently create a biplanar spinal deformity confirming alterations in spinal growth (18). Using an ultrahigh molecular weight polyethylene (UHMWPE) anterolateral spinal tether in a mini-pig model demonstrated the creation of vertebral body wedging in a tethered group of $14^{\circ}$ after 6 months and $30^{\circ}$ after 12 months, compared to a surgical sham group in which no deformity was created (19). In 2005, Braun et al. (20) in an experimental scoliosis model in goats demonstrated improvement in scoliosis after flexible tethers were placed.

Intervertebral disc health has also been measured following anterior vertebral body tethers; a future treatment strategy needs to maintain the intervertebral disc function if it is to be a successful long-term method for fusionless scoliosis. In the mini-pig model, the vertebral body became wedged, convex on the side of the tether, and was associated with wedging in the discs in an opposite manner (convex on the contralateral side of the tether). Disc samples from spines following tethering were found to be well hydrated, with MR T2 signal similar to sham surgical discs $(19,21)$. Fusionless scoliosis implants have been found to result in alterations in intervertebral disc cell density (22) and collagen content (23), however the clinical implications of these changes are unknown.

The mechanism by which application of compressive forces acts to modulate the growth of vertebral bodies is thought to be at the level of the vertebral growth plate. Chay et al. found that tethering an experimentally created scoliotic curve in pigs resulted in a decrease in proliferative zone height of the growth plate on the side of tether compression compared to the opposite side of the vertebrae (24). The hypertrophic zone height and cartilage cell numbers within this zone are reduced following a unilateral application of compression on the vertebral body $(25,26)$.

\section{Clinical evidence}

The specific indications for attempting to correct scoliosis via a growth modulation strategy are evolving as we gain greater clinical experience. The ideal patient is likely a preadolescent with an idiopathic etiology and a scoliosis $>45^{\circ}$ that has a high likelihood of curve progression due to remaining growth. Our current indications to consider anterior vertebral growth modulation with tethering are patients with primary thoracic curves of 45-65 degrees and Risser 0 or 1, Sanders 3-4 (Figure 1). The thoracic spine should be hypokyphotic. Indications for tethering thoracolumbar curves remain unclear, although this is an attractive region to attempt to spare fusion, the durability of current implant options remains a concern. As with all surgery that can have an effect related to growth, close monitoring is mandatory as there is a risk of overcorrection and the consequences can create a substantial deformity on the opposite direction (Figure 2).

There clinical experience and peer reviewed publications on anterior vertebral growth modulation remain limited. The first case report came from Crawford and Lenke (27) in 2010. They presented a case of thoracic curve tethering with clear evidence of growth modulation in an 8-year-old male. Shortly after this, several surgeons began using devices "off label" and subsequent experience resulted in 2 publications by Samdani et al. in 2014 (28) and 2015 (29). The first reported on 11 patients with an average age of $12 \pm 3$ years and 2 years of follow-up. The preoperative 

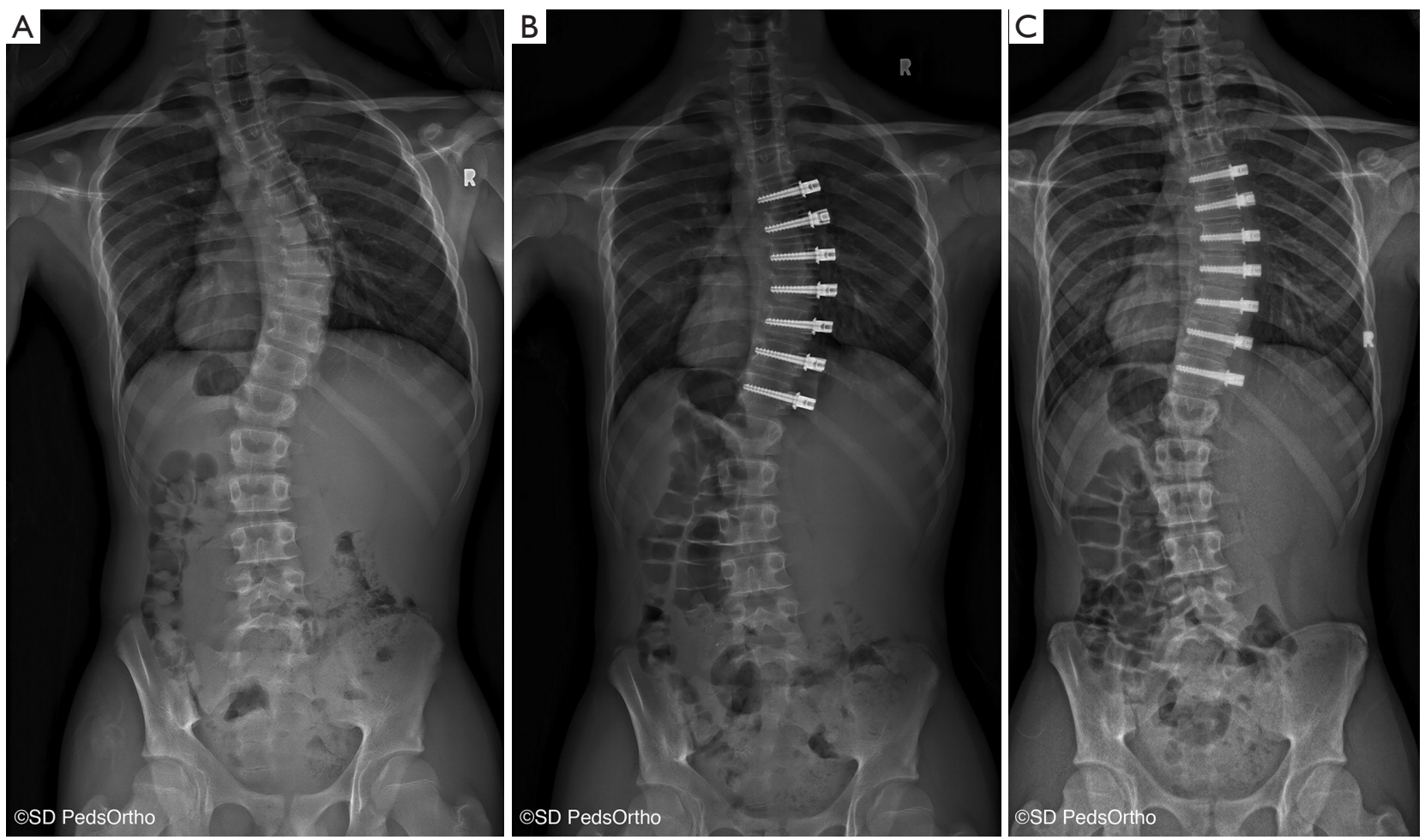

Figure 1 (A) A 15-year-old male who was Risser 1, Sanders 3 at the time of tether placement; (B) immediate postop correction; (C) 3 years postop he completed growth with satisfactory alignment.

curve of $44 \pm 9$ degrees was improved to $14 \pm 12$ degree with an $18 \%$ revision rate ( 2 cases of loosening the tether due to overcorrection). This was followed by a larger series of 32 patient with similar correction of $43 \pm 8$ degree to $18 \pm 11$ degrees at 1 -year follow-up. Cobetto et al. (30) published a series of 20 patients with 2 years of followup with correction from $59 \pm 10$ degree to $23 \pm 8$ degrees. Newton et al. (31) also published in 2018 a series of 17 thoracic tethering procedures with 2- to 4-year followup. The average age of the patients was 11.2 years and 16 of the 17 had open triradiate cartilage. The average preoperative curve magnitude was $52 \pm 10$ degrees and was corrected by $51 \%$ to $27 \pm 20$ degrees at latest follow-up. Seven patients underwent a revision procedure, four due to overcorrection, a 3 for progressive deformity (1 posterior fusion, 1 replacement of a broken tether and 1addition of a lumbar tether). A curve at latest follow-up of less than 35 degree was achieved in 10 of the 17 patients. This paper also identified evidence of tether breakage based on increasing screw divergence over time in some cases, confirming implant failure over time as a concern.

The number of unpublished reports being presented at international meetings has been increasing in the past several years. The follow-up remains short and the indications with regards to curve size, location and growth remaining at the time of surgery remain quite variable. One notable presentation at 2019 annual meeting of the Scoliosis Research Society was the presentation by Samdani et al. (32) on their series that was used to support the FDA application for approval of a tether device. A series of 53 patient of Lenke type $1 \mathrm{~A}$ and $1 \mathrm{~B}$ curves with a mean follow-up of 4 years was presented. The average curve magnitude preoperatively (40 degrees) was noted to smaller than the authors' current indications and the most recent postoperative deformity averaged 16 degrees. There were 5 revisions and $86 \%$ had a curve of $<30$ degrees at last follow-up.

\section{Intraoperative considerations}

The authors prefer a thoracoscopic approach for tether insertion, although open and "mini" open approaches are also possible. Basic thoracoscopy skills are required in order to safely navigate the chest cavity. A general/thoracic surgeon with thoracoscopic experience can greatly easy 

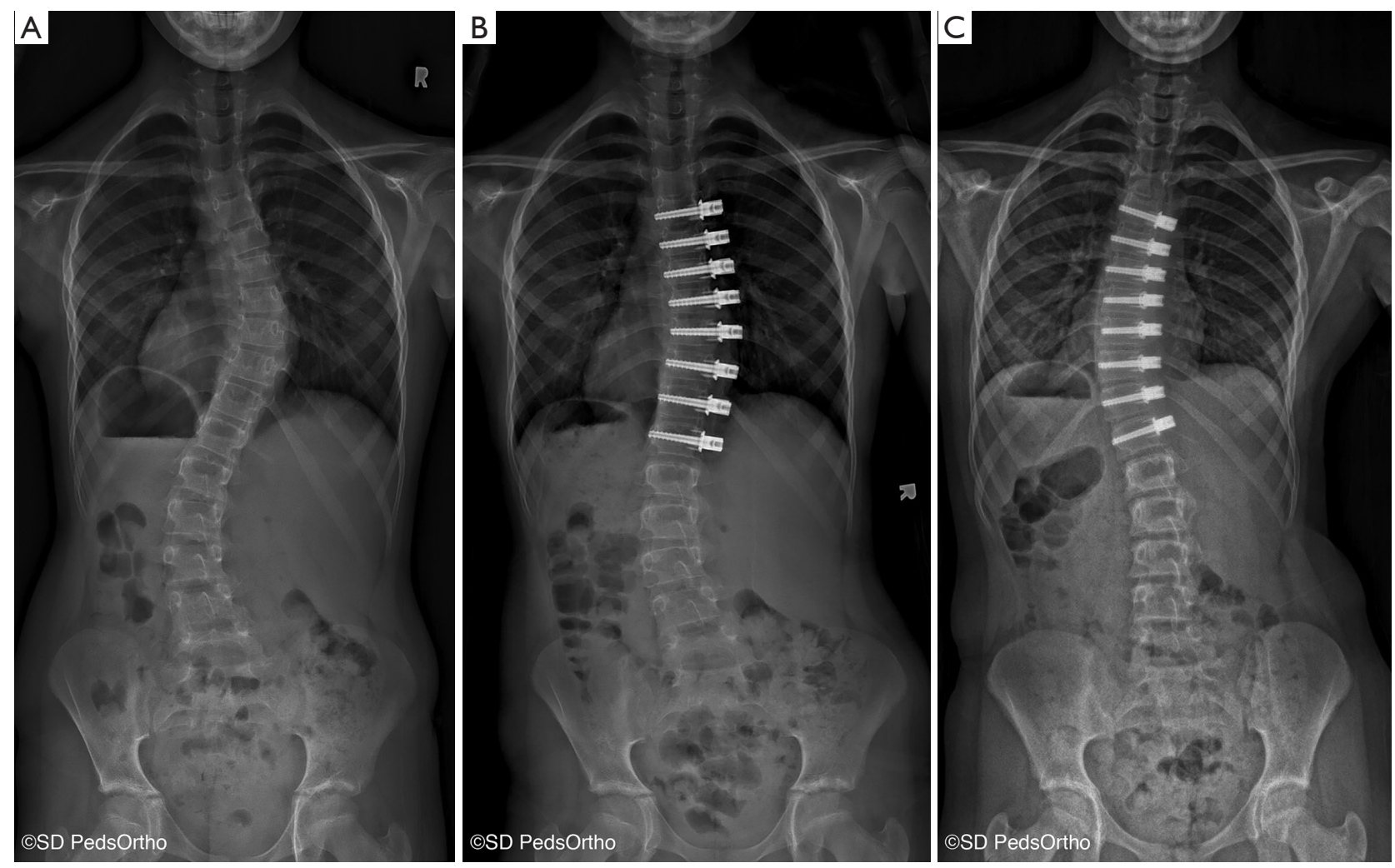

Figure 2 (A) A 11-year-old female who was Risser 0 with open triradiate cartilage at the time of tether placement; (B) immediate postop correction; (C) 2 years postop she developed moderate over correction and underwent revision surgery to release the implant cord.

the learning curve of such a procedure. The technique of performing thoracoscopic anterior spinal instrumentation has been previously described for fusion and these principles apply to tethering as well (33-36). Briefly, the lungs are selectively ventilated (with a double lumen endotracheal tube) allowing the convex lung to collapse opening a large space in the chest to safely access the anterior spine. As with thoracoscopic instrumentation for fusion, three $15 \mathrm{~mm}$ ports are placed along the posterior axillary line in positions that will allow direct lateral access for screw placement. A single anterior axillary line portal at the apex of the curve is used for scope placement. The pleura is incised longitudinally and the segmental vessels coagulated and divided using ultrasonic energy.

A biocritical screw path is created in the mid portion of each vertebral body and screw length chosen without excessive screw penetration that may put the aorta at risk. Pronged washers are used to reinforce the lateral cortex and reduce the risk of screw levering/plowing over time. Screws are placed into each vertebra included in the thoracic scoliosis deformity (from upper end vertebra to lower end vertebra). The image intensifier, intraoperative CT or a navigation system may be utilized to confirm trajectory and screw length. With the screws placed, the PET cord is tensioned between the screws and locked into position. The tension in the cord can be varied utilizing an external tensioning device depending on the deformity and the growth remaining of the patient. An attempt to bring the wedged discs (thinner on concave side and thicker on convex side of the scoliotic spine) to parallel without reversing the wedging may the best criteria for tension application inter-operatively. Less tension is required at the ends of the construct as less deformity is present in these segments. Caution is advised against over tensioning as this may be both detrimental to disc physiology as well as lead to rapid over correction particularly at the distal end where growth is the fastest.

\section{Authors indications}

The indications for anterior spinal tethering procedures 
remain controversial and with the limited evidence available are likely to remain so for some time to come. My very strong conviction is that this technique was entirely based on the principle of spinal growth modulation and thus in my view "substantial" growth must remain to lead to enough deformity correction to warrant a surgical procedure. Growth remaining is unfortunately not precisely predictable, neither in absolute time nor total centimeters. We estimate skeletal age based on radiographic markers (pelvis, hand, shoulder, elbow) with the most well know being the Risser sign. Although convenient as it is included on the spine radiograph it is not the best predictor of remaining growth. Sanders has popularized the simplified scoring system based on the hand radiograph (37). These stages are more reliably related to the percentage of total height gain remaining (38). My preference is to consider patients who have a Sanders score of 3 or 4 as those with the "ideal" remaining growth for tethering patients with thoracic scoliosis. This is unfortunately based on very little evidence.

With regards to curve location and magnitude, I prefer right thoracic curves of 45-65 degrees. For curves less than this I prefer a course of bracing (39) and larger curves have been less reliably controlled with a tether and I prefer spinal fusion. The size of the secondary curves certainly plays into the selection of an ideal candidate as well. As the upper thoracic and lumbar curves increase the concept of an isolated thoracic tethering procedure becomes less attractive. An upper thoracic curve with acceptable shoulder appearance can be considered. Similarly for the lumbar region and flexible $\mathrm{C}$ modifier curves can be considered although Lenke AL and B curves are best. The AR curve pattern has been problematic with adding on, just as it may in cases treated with fusion. Tethering more than one curve likely reduces the predictability of the procedure's overall outcome. I have limited the vast majority of my personal early tethering experience to thoracic curves, despite the potential for even greater benefit to sparing motion in the lumbar spine. We do have a limited experience combining selective thoracic fusion with contralateral lumbar tethering for Lenke $1 \mathrm{C}$ and $3 \mathrm{C}$ curves thought not amenable to selective thoracic fusion alone. I fear lumbar tether implant breakage will occur at rates even higher than we have seen in the thoracic region.

I do not believe patients with limited growth remaining or those that have completed growth are appropriate for implantation of these devices. Immediate correction through the mobility of the discs is certainly achievable intraoperatively, but without substantial 3-dimensional shape change of the vertebral bodies (via growth) possible, I do not believe we have created a circumstance that will be maintainable for the long term. Depending on the implant for long-term success is unwise. The skeletal systems mechanisms for adapting to alteration in the loading environment certainly exist in bone and soft tissues, however we have no preclinical data to suggest use of a tether in the skeletally mature spine can result in permanent changes in shape.

Ultimately the patient and family will need to assess their own preferences and values in considering whether a tethering approach is appropriate. We may guide patients who are considered candidates with the available evidence (as limited as it is) in comparison to standard posterior spinal fusion approaches for which there is extensive evidence to inform the risks and expected outcomes. Patients often over-estimate the loss of mobility and function associated with a thoracic spinal fusion and under-estimate the challenges associated with "minimally invasive" spinal surgery. Education and evidence generation are required to inform the shared decision-making process.

\section{Conclusions}

There is a growing body of evidence that spinal growth can be modulated with anterior vertebral body tethering devices in growing patients with scoliosis. The basic science and experimental studies support the premise that the asymmetric mechanical compression of vertebral body growth centers (endplates) can slow growth on the anterior and convex aspect of the spinal column. The early clinical experience also confirms the potential and several publications of the early outcomes demonstrate average reductions in scoliosis magnitude over time with growth. Despite the promising potential there remains much to learn regarding the precise indications and the patient specific constructs required give each patients curve pattern and remaining skeletal growth potential. Intervening too early or too aggressively may result in overcorrection and implanting the device in too large a curve or too late in the growth spurt may not provide adequate change in vertebral shape to produce a lasting correction. We should not anticipate that long term correction can be maintained by the device alone as cord failure with currently available materials is common after $2-3$ years.

\section{Acknowledgments}

None. 


\section{Footnote}

Conflicts of Interest: The author has no conflicts of interest to declare.

Ethical Statement: The author is accountable for all aspects of the work in ensuring that questions related to the accuracy or integrity of any part of the work are appropriately investigated and resolved.

\section{References}

1. Braun JT, Ogilvie JW, Akyuz E, et al. Fusionless scoliosis correction using a shape memory alloy staple in the anterior thoracic spine of the immature goat. Spine (Phila Pa 1976) 2004;29:1980-9.

2. Cheh G, Bridwell KH, Lenke LG, et al. Adjacent segment disease followinglumbar/thoracolumbar fusion with pedicle screw instrumentation: a minimum 5-year followup. Spine (Phila Pa 1976) 2007;32:2253-7.

3. Danielsson AJ, Cederlund CG, Ekholm S, et al. The prevalence of disc aging and back pain after fusion extending into the lower lumbar spine. A matched MR study twenty-five years after surgery for adolescent idiopathic scoliosis. Acta Radiol 2001;42:187-97.

4. Hilibrand AS, Robbins M. Adjacent segment degeneration and adjacent segment disease: the consequences of spinal fusion? Spine J 2004;4:190S-4S.

5. Lerner T, Frobin W, Bullmann V, et al. Changes in disc height and posteroanterior displacement after fusion in patients with idiopathic scoliosis: a 9-year follow-up study. J Spinal Disord Tech 2007;20:195-202.

6. Park P, Garton HJ, Gala VC, et al. Adjacent segment disease after lumbar or lumbosacral fusion: review of the literature. Spine (Phila Pa 1976) 2004;29:1938-44.

7. Nachlas IW, Borden JN. The cure of experimental scoliosis by directed growth control. J Bone Joint Surg Am 1951;33 A:24-34.

8. Braun JT, Ogilvie JW, Akyuz E, et al. Experimental scoliosis in an immature goat model: a method that creates idiopathic-type deformity with minimal violation of the spinal elements along the curve. Spine (Phila Pa 1976) 2003;28:2198-203.

9. Trobisch PD, Samdani A, Cahill P, et al. Vertebral body stapling as an alternative in the treatment of idiopathic scoliosis. Oper Orthop Traumatol 2011;23:227-31.

10. Lavelle WF, Samdani AF, Cahill PJ, et al. Clinical outcomes of nitinol staples for preventing curve progression in idiopathic scoliosis. J Pediatr Orthop 2011;31:S107-13.

11. Braun JT, Hoffman M, Akyuz E, et al. Mechanical modulation of vertebral growth in the fusionless treatment of progressive scoliosis in an experimental model. Spine (Phila Pa 1976) 2006;31:1314-20.

12. Betz RR, Kim J, D'Andrea LP, et al. An innovative technique of vertebral body stapling for the treatment of patients with adolescent idiopathic scoliosis: a feasibility, safety, and utility study. Spine (Phila Pa 1976) 2003;28:S255-65.

13. Betz RR, D'Andrea LP, Mulcahey MJ, et al. Vertebral body stapling procedure for the treatment of scoliosis in the growing child. Clin Orthop Relat Res 2005;(434):55-60.

14. Guille JT, D'Andrea LP, Betz RR. Fusionless treatment of scoliosis. Orthop Clin North Am 2007;38:541-5, vii.

15. Braun JT, Hines JL, Akyuz E, Vallera C, Ogilvie JW. Relative versus absolute modulation of growth in the fusionless treatment of experimental scoliosis. Spine (Phila Pa 1976) 2006;31:1776-82.

16. Aronsson DD, Stokes IA, Rosovsky J, et al. Mechanical modulation of calf tail vertebral growth: implications for scoliosis progression. J Spinal Disord 1999;12:141-6.

17. Newton PO, Fricka KB, Lee SS, et al. Asymmetrical flexible tethering of spine growth in an immature bovine model. Spine (Phila Pa 1976) 2002;27:689-93.

18. Newton PO, Faro FD, Farnsworth CL, et al. Multilevel spinal growth modulation with an anterolateral flexible tether in an immature bovine model. Spine (Phila Pa 1976) 2005;30:2608-13.

19. Upasani VV, Farnsworth CL, Chambers RC, et al. Intervertebral disc health preservation after six months of spinal growth modulation. J Bone Joint Surg Am 2011;93:1408-16.

20. Braun JT, Akyuz E, Ogilvie JW, et al. The efficacy and integrity of shape memory alloy staples and bone anchors with ligament tethers in the fusionless treatment of experimental scoliosis. J Bone Joint Surg Am 2005;87:2038-51.

21. Newton PO, Upasani VV, Farnsworth CL, et al. Spinal growth modulation with use of a tether in an immature porcine model. J Bone Joint Surg Am 2008;90:2695-706.

22. Hunt KJ, Braun JT, Christensen BA. The effect of two clinically relevant fusionless scoliosis implant strategies on the health of the intervertebral disc: analysis in an immature goat model. Spine (Phila Pa 1976) 2010;35:371-7.

23. Newton PO, Farnsworth CL, Faro FD, et al. Spinal 
growth modulation with an anterolateral flexible tether in an immature bovine model: disc health and motion preservation. Spine (Phila Pa 1976) 2008;33:724-33.

24. Chay E, Patel A, Ungar B, et al. Impact of Unilateral Corrective Tethering on the Histology of the Growth Plate in an Established Porcine Model for Thoracic Scoliosis. Spine (Phila Pa 1976) 2012;37:E883-9.

25. Bylski-Austrow DI, Wall EJ, Glos DL, et al. Spinal hemiepiphysiodesis decreases the size of vertebral growth plate hypertrophic zone and cells. J Bone Joint Surg Am 2009;91:584-93.

26. Newton PO, Glaser DA, Doan JD, et al. 3D Visualization of Vertebral Growth Plates and Disc: The Effects of Growth Modulation. Spine Deformity, 2013;1:313-20.

27. Crawford CH 3rd, Lenke LG. Growth modulation by means of anterior tethering resulting in progressive correction of juvenile idiopathic scoliosis: a case report. J Bone Joint Surg Am 2010;92:202-9.

28. Samdani AF, Ames RJ, Kimball JS, et al. Anterior vertebral body tethering for idiopathic scoliosis: two-year results. Spine (Phila Pa 1976) 2014;39:1688-93.

29. Samdani AF, Ames RJ, Kimball JS, et al. Anterior vertebral body tethering for immature adolescent idiopathic scoliosis: one-year results on the first 32 patients. Eur Spine J 2015;24:1533-9.

30. Cobetto N, Aubin CE, Parent S. Surgical Planning and Follow-up of Anterior Vertebral Body Growth Modulation in Pediatric Idiopathic Scoliosis Using a Patient-Specific Finite Element Model Integrating Growth Modulation. Spine Deform 2018;6:344-350.

31. Newton PO, Kluck DG, Saito W, et al. Anterior Spinal

Cite this article as: Newton PO. Spinal growth tethering: indications and limits. Ann Transl Med 2020;8(2):27. doi: 10.21037/atm.2019.12.159
Growth Tethering for Skeletally Immature Patients with Scoliosis: A Retrospective Look Two to Four Years Postoperatively. J Bone Joint Surg Am. 2018;100:1691-7.

32. Samdani A, Pahys J, Ames RJ et al. Prospective Followup o Anterior Vertebral Body Tethering (AVBT) for idiopathic scoliosis: Interim results from an FDA IDE study. Montreal, Canada: Scoliosis Research Society Annual Meeting, 2019.

33. Newton PO, Upasani VV, Lhamby J, et al. Surgical treatment of main thoracic scoliosis with thoracoscopic anterior instrumentation. Surgical technique. J Bone Joint Surg Am 2009;91 Suppl 2:233-48.

34. Lonner BS. Emerging minimally invasive technologies for the management of scoliosis. Orthop Clin North Am 2007;38:431-40; abstract vii-viii.

35. Dickman C, Rosenthal D, Perin N. Thoracoscopic Spine Surgery. Vol. 1. New York: Theime Medical Publishers, Inc., 1999.

36. Frantzides CT. Laparoscopic and Thoracoscopic Surgery. St. Louis: Mosby-Year Book, Inc., 1995.

37. Sanders JO, Khoury JG, Kishan S, et al. Predicting scoliosis progression from skeletal maturity: a simplified classification during adolescence. J Bone Joint Surg Am 2008;90:540-53.

38. Sanders JO, Qiu X, Lu X, et al. The Uniform Pattern of Growth and Skeletal Maturation during the Human Adolescent Growth Spurt. Sci Rep 2017;7:16705.

39. Weinstein SL, Dolan LA, Wright JG, et al. Effects of bracing in adolescents with idiopathic scoliosis. N Engl J Med 2013;369:1512-21. 